Revue des sciences de l'éducation

\title{
Articles de revues parus en 1984 dans le domaine de la sociologie de l'éducation
}

\section{Claude Trottier}

Volume 11, numéro 1, 1985

URI : https://id.erudit.org/iderudit/900489ar

DOI : https://doi.org/10.7202/900489ar

Aller au sommaire du numéro

Éditeur(s)

Revue des sciences de l'éducation

ISSN

0318-479X (imprimé)

1705-0065 (numérique)

Découvrir la revue

Citer ce document

Trottier, C. (1985). Articles de revues parus en 1984 dans le domaine de la sociologie de l'éducation. Revue des sciences de l'éducation, 11(1), 193-210. https://doi.org/10.7202/900489ar d'utilisation que vous pouvez consulter en ligne. 


\section{Articles de revues parus en 1984 dans le domaine de la sociologie de l'éducation ${ }^{1}$}

Les études portant sur les fondements sociaux de l'éducation, et plus particulièrement sur la sociologie de l'éducation, en sont venues à occuper une place importante dans le développement des sciences de l'éducation des vingt dernières années. Dans quelle direction les études et les recherches actuelles sont-elles orientées? Quelles sont les préoccupations sous-jacentes aux travaux qui ont été publiés récemment? C'est dans ce contexte et par rapport à ces questions qu'il est apparu pertinent de procéder à une recension d'articles de revues parues en 1984 dans le domaine de la sociologie de l'éducation.

L'objectif de cette recension n'est pas de présenter un résumé de tous les articles de sociologie de l'éducation qui seraient parus dans diverses revues au cours de l'année. La "production " est telle qu'il serait irréaliste d'y penser. L'objectif n'est pas non plus de faire une analyse de contenu systématique de ces articles pour dégager les tendances de la sociologie de l'éducation. Celles-ci ne sauraient être reconstituées sur la base d'une seule année. Il est encore moins de faire une critique de ces travaux. L'objectif est plutôt de rendre compte d'articles qui revêtent un intérêt particulier soit parce qu'ils contribuent à renouveler les problématiques ou à présenter des faits nouveaux sur des problèmes qui s'inscrivent dans les domaines traditionnels de recherche de la sociologie de l'éducation, soit parce qu'ils reflètent de nouvelles orientations au sein de la discipline.

Cette recension aura forcément un caractère sélectif et ne visera pas à être exhaustive. Les articles qui ont été retenus pour commentaires ont été sélectionnés soit parce qu'ils sont liés à des problèmes auxquels la sociologie de l'éducation s'est intéressée depuis un certain temps, soit parce qu'ils ont trait à des questions qui ont été jugées pertinentes dans le contexte des années 80 ou dans le contexte québécois et canadien, soit parce que plusieurs articles de revues diverses portent sur un même thème, soit parce que des revues ont publié des numéros spéciaux sur des thèmes 
spécifiques. Il y a donc lieu de reconnaître d'emblée ce que cette sélection peut avoir d'arbitraire. Par ailleurs, il est possible d'identifier dans la "production" de l'année 1984 des convergences qu'on ne saurait ignorer, et qui font que certains thèmes s'imposent à l'attention lorsqu'on jette un regard sur un ensemble d'articles parus au cours de l'année.

Le lecteur aura un aperçu des revues qui ont été consultées à partir de la liste des références. Il se rappellera toutefois que plusieurs autres revues que celles qui apparaissent dans cette liste ont été recensées, et que des articles n'ont pas été sélectionnés dans toutes les revues consultées. Il aura compris de plus que la recension ne porte pas seulement sur des articles parus dans les revues de sociologie de l'éducation proprement dites. Il aurait fallu dans ce cas nous limiter à deux revues, Sociology of Education et British Journal of Sociology of Education. Nous avons donc choisi des articles parus dans des revues de sciences sociales et de sciences de l'éducation, de langue française et de langue anglaise.

Les articles sélectionnés ont été regroupés autour des thèmes suivants: les facteurs sociaux de l'apprentissage, le processus de socialisation, les travaux ayant une portée théorique, les travaux ayant une portée historique, l'insertion socioprofessionnelle des jeunes, femmes et éducation, l'analyse de phénomènes internes à l'institution scolaire, les savoirs scolaires et les programmes, les études ethnographiques.

\section{Les facteurs sociaux de l'apprentissage}

L'influence des facteurs sociaux sur la réussite et les cheminements scolaires des étudiants est un thème qui occupe depuis longtemps une place de premier plan dans la discipline. Les sociologues de l'éducation ont en effet été à la fois soucieux et pressés de démontrer qu'ils pouvaient projeter un éclairage différent de celui des psychopédagogues sur l'apprentissage des élèves. Quatre articles relatifs à ce thème feront l'objet d'un commentaire.

Alwin et Thornton (1984) ont tenté de circonscrire l'effet du statut socioéconomique des parents sur la réussite, le cheminement scolaire et le nombre d'années de scolarité des élèves. Cet article est intéressant pour plusieurs raisons. On y présente les résultats d'une recherche longitudinale qui a porté sur une période de 18 ans (1962 à 1980). Pour mesurer la position socio-économique des parents, on a fait appel à des indicateurs traditionnels (niveau de scolarité du père et de la mère, occupation du père, revenu de la famille) mais aussi à des indicateurs qui avaient été peu utilisés jusqu'à présent (possession de biens immobiliers et de capital de la famille, participation de la mère au marché du travail, dimension de la famille). Enfin, les auteurs essaient de mesurer l'influence du statut socio-économique de la famille à divers moments de la carrière scolaire des élèves. Il ressort de l'analyse que, quel que soit le moment où on mesure le statut socio-économique de la famille, l'influence de ce dernier sur le cheminement scolaire et le développement cognitif des étudiants 
demeure constante, bien qu'il y ait des indications à l'effet qu'elle soit plus forte au début de la carrière scolaire qu'aux autres étapes de celle-ci.

Hearn (1984) pour sa part discute de l'influence relative des facteurs académiques (résultats à différents tests d'aptitude) et des facteurs liés à l'origine sociale (classe sociale, race et sexe) des étudiants sur le type d'université (niveau plus ou moins élevé de prestige ou de qualité de l'institution) qu'ils fréquentent. L'auteur arrive à la conclusion que l'effet de l'origine sociale des étudiants sur le type d'université qu'ils fréquentent n'est pas très important même si la relation entre les deux variables demeure significative sur le plan statistique. Les étudiants d'origine sociale inférieure auront moins tendance à fréquenter les universités qui requièrent des frais de scolarité élevés et qui mettent plus de ressources à la disposition des étudiants. Ils auront aussi moins tendance à fréquenter les universités qui ont des pratiques d'admission très sélectives. L'effet de l'origine sociale dans ce dernier cas cependant est médiatisé par des facteurs académiques, c'est-à-dire les étudiants d'origine sociale supérieure ont accès en plus grand nombre que ceux d'origine sociale inférieure aux universités les plus sélectives sur le plan académique à condition qu'ils aient d'abord un rendement académique supérieur. Ces résultats mettent en relief que la démocratisation de l'accès à l'université pourrait avoir un effet limité sur l'égalisation des chances, si une stratification en terme de prestige et de qualité des institutions ou des programmes d'étude est maintenue. Dans cette perspective, les étudiants d'origine sociale inférieure n'auraient accès qu'aux universités et aux programmes dont la qualité et le prestige sont les moins élevés.

La fréquentation d'une école publique ou d'une école privée peut-elle avoir un effet sur la poursuite d'études post-secondaires et sur le type d'institution postsecondaire fréquentée? Telle est la question que se posent Falsey et Heyns (1984). L'analyse a été conduite à partir d'un échantillon d'étudiants américains interviewés dans le cadre d'une recherche longitudinale. Il ressort de l'analyse que les élèves fréquentant une école privée ont davantage tendance que les étudiants fréquentant une école publique à poursuivre des études post-secondaires et à s'inscrire dans des institutions d'enseignement post-secondaire qui dispensent des programmes d'une durée de quatre ans plutôt que dans des institutions d'enseignement post-secondaire qui offrent des programmes plus courts. Ces tendances se maintiennent même lorsque les chercheurs contrôlent l'effet d'autres variables comme la section de l'école secondaire ("TRACK») à laquelle appartiennent les étudiants, leur niveau d'habilité académique, leur origine sociale et leurs aspirations. Les auteures ne manquent pas l'occasion de prendre position dans le débat sur l'influence relative des facteurs scolaires et de l'origine sociale des élèves sur leur cheminement. Selon elles, le type d'école fréquentée par les élèves peut avoir un impact sur leur carrière scolaire. Elles suggèrent que cet effet peut être lié aux politiques organisationnelles de l'école, aux orientations du personnel, aux ressources et aux modalités d'encadrement qu'on met à la disposition des élèves susceptibles de poursuivre des études post-secondaires. 
Crespo et Pelletier (1984) essaient de circonscrire l'impact des classes d'accueil sur le rendement scolaire des enfants d'immigrants dans la région de Montréal. Cet article revêt un intérêt particulier dans le contexte québécois et canadien. Les auteurs y présentent les résultats d'une recherche longitudinale sur l'effet des classes d'accueil sur cette clientèle scolaire, une fois que celle-ci est intégrée aux classes régulières.

\section{Le processus de socialisation}

L'école ne fait pas que transmettre des connaissances et son rôle ne se limite pas uniquement au développement cognitif des élèves. Elle transmet aussi des normes, des valeurs et des idéologies. Elle participe à la socialisation des jeunes générations. La sociologie de l'éducation continue d'accorder beaucoup d'importance à ce thème, comme en témoignent les articles suivants. On a longtemps pensé que le seul fait de scolariser davantage les jeunes générations pouvait contribuer à diminuer l'intolérance et les préjugés, et susciter un attachement plus grand à des orientations démocratiques. Dans une étude effectuée aux États-Unis à partir de données tirées d'un sondage, Jackman et Muha (1984) ont tenté de circonscrire l'effet de la scolarisation des individus sur ces attitudes. Les résultats de leur analyse les amènent à remettre en question plusieurs des idées reçues sur cette question. Les attitudes négatives à l'égard des minorités ne semblent pas diminuer, et l'adhésion aux valeurs démocratiques ne semble pas augmenter avec le niveau de scolarité. Selon eux, l'éducation contribuerait plutôt à placer les individus les plus scolarisés en avance de leurs pairs qui le sont peu, dans la défense de leurs propres intérêts.

Harber (1984) pose jusqu'à un certain point le même problème mais dans une perspective et un contexte différent. Dans un pays en voie de développement, quel effet la scolarisation peut-elle avoir sur l'apprentissage des valeurs démocratiques et d'autres attitudes requises par le développement économique et par les changements qui se produisent dans la structure sociale? L'auteur s'emploie à démontrer qu'il n'est pas suffisant d'augmenter le niveau de scolarité des individus. Selon lui, il importe non seulement de transmettre de façon explicite aux jeunes les valeurs démocratiques, qu'on se propose de leur inculquer, mais aussi de les socialiser aux divers rôles sociaux qui en découlent. Deux autres articles relatifs à ce thème posent non plus le problème de l'effet de la scolarisation sur les attitudes politiques, mais celui de certaines variables sur l'apprentissage des normes et des valeurs politiques. Passeron et De Singly (1984) présentent une analyse de plusieurs représentations et pratiques des jeunes (niveau de connaissance dans divers domaines, autonomie par rapport aux parents, rôles sexuels, sexualité, contestation de la société, intérêt pour la politique). Cet article est intéressant parce a) que les auteurs ne se sont pas confinés à des représentations ou pratiques étroitement politiques comme plusieurs études sur la socialisation politique ont tendance à le faire, b) que leurs résultats portent sur trois classes d'âge dans trois pays d'Europe, c) qu'ils essaient de cerner l'effet de l'appartenance à une classe sociale et du sexe sur ces représentations et pratiques. Ils 
démontrent que celles-ci varient d'abord en fonction de la classe sociale d'origine, mais aussi en fonction du sexe. Ils essaient d'évaluer jusqu'à quel point une classe sociale "spécialise " l'éducation des filles et des garçons, pour ensuite mettre en relief les proximités et les distances entre classes sociales sous le rapport de l'homogénéité de leur socialisation sexuelle.

Close (1984) pour sa part pose le problème de la socialisation des jeunes aux affaires internationales à partir de données recueillies auprès de trois groupes d'étudiants terre-neuviens d'âges différents (cinq, huit et onze ans). L'intérêt de l'article tient à ce que peu d'études ont été effectuées sur cet aspect de la socialisation politique (et encore moins dans un contexte canadien). L'auteur essaie de mesurer les connaissances que les jeunes ont des affaires internationales et de circonscrire l'effet de certaines variables sur ce type de connaissance. Il conclut que les jeunes sont légèrement plus informés des affaires nationales que des affaires internationales, et que le niveau de ces deux types de connaissance varie en fonction des mêmes variables, soit l'âge, le sexe et la classe sociale d'origine.

Ces quatre articles sur la socialisation des jeunes renvoient dans une certaine mesure au problème de l'éducation politique des jeunes. Il y aurait lieu à cet égard de porter à l'attention des lecteurs un numéro spécial de la revue britannique Education Review (vol. 36, no 2) sur l'éducation politique. Tous les articles de ce numéro n'ont pas nécessairement été conçus dans une perspective sociologique mais plusieurs d'entre eux présentent un intérêt certain, notamment ceux portant sur la politique et l'éducation politique, l'enseignement relatif à des problèmes controversés, le développement de concepts et d'habilités politiques à l'élémentaire, l'identité nationale et les affaires internationales, la socialisation politique des jeunes noirs et des femmes.

\section{Les travaux ayant une portée théorique}

Nous tenterons tout d'abord de rendre compte d'un ensemble d'articles qui ont trait, à des degrés divers, aux débats qui entourent les théories de la reproduction en éducation.

On sait, en effet que ces théories sont fortement remises en question. C'est à propos des fonctions de la formation continue en France que Dubar (1984) pose le problème. Contribue-t-elle d'abord au développement des capacités des individus? Jusqu'à quel point cette formation peut-elle être définie comme un investissement dans leur "capital humain " ? Ou au contraire, n'est-elle qu'un outil de gestion dont dispose le patronnat, et un instrument de reproduction sociale? Dubar estime que la formation continue ne peut être vue comme une simple instance de reproduction de. rapports sociaux de type capitaliste. Elle peut aussi permettre une appropriation progressive des éléments culturels nécessaires à la compréhension et à la trar rírmation de la situation sociale des individus qui s'y engagent.

Shapiro (1984a) remet aussi très nettement en question la façon de 
conceptualiser l'école comme un instrument de reproduction, de domination et de répression qui se dégage de certaines théories de la reproduction. Selon lui, l'image simpliste qui se dégage de l'école et des élèves dans certaines de ces théories est celle d'une manufacture qui "produit" des élèves passifs dont les orientations correspondent aux attentes du système de production. La critique qu'il en fait s'inspire du concept d'hégémonie de Gramsci et d'une analyse de la domination culturelle différente de celle qui avait été proposée par les premiers théoriciens de la reproduction en éducation. L'auteur propose une conception de l'école a) qui rende compte du caractère conservateur de celle-ci sans verser dans une approche conspirationniste de la socialisation, et b) qui fait place à une analyse plus raffinée du contexte institutionnel de l'école et du fonctionnement interne du système d'enseignement.

Ninalowo (1984) s'efforce aussi de montrer que le système d'enseignement ne remplit pas qu'une fonction de reproduction et de légitimation de l'ordre établi. L'auteur estime que ce postulat est sous-jacent aussi bien au paradigme fonctionnel qu'au paradigme radical. Il fait valoir que le système d'enseignement peut aussi être un instrument de conscientisation, que les élèves peuvent intérioriser de façon sélective les normes et les valeurs véhiculées par le système d'enseignement. Il est permis de s'interroger cependant sur le rapprochement qu'il fait à cet égard entre le paradigme fonctionnel et le paradigme radical. Il y aurait lieu de mieux situer les deux paradigmes l'un par rapport à l'autre, et de montrer aussi en quoi ils mettent de l'avant des conceptions très différentes de l'orientation conservatrice du système d'enseignement.

Gordon (1984), pour sa part, fait ressortir les éléments qui, dans les travaux de Willis $(1979,1983)$, permettent de dépasser une conception trop mécanique de la reproduction. Les orientations proposées par Willis débouchent, selon lui, sur une meilleure articulation des relations entre éducation, culture et idéologie, et permettent de reconnaître les possibilités de transformation et de changement que présente la production culturelle à l'intérieur du système d'enseignement. Harker (1984), quant à lui, prend la défense de Bourdieu contre les critiques de ce dernier. Il tente de montrer que sa conception de la reproduction et en particulier sa théorie de la pratique, contrairement à ce qu'on a souvent dit des travaux de Bourdieu, font une place au changement, de même qu'aux stratégies et à l'initiative des acteurs dans ce processus.

Ces articles sur les théories de la reproduction en éducation témoignent d'une remise en question systématique de ces théories par des auteurs qui s'inspirent d'autres perspectives théoriques ou même de variantes des théories de la reproduction elle-même. De plus, il ressort que même les auteurs qui en prennent la défense, ont tendance à proposer des réorientations dans le but d'en faire des instruments conceptuels qui seraient plus en mesure de rendre compte du changement et de l'initiative des acteurs sociaux dans la production et la reproduction culturelle.

Au-delà des articles qui nourissent le débat sur les théories de la repro- 
duction, il y a lieu de mentionner d'autres articles de portée théorique qui revêtent un intérêt particulier. Shapiro (1984b) propose une réflexion sur la crise qui secoue le monde de l'éducation au cours des années 80 . Pourquoi est-on moins convaincu qu'auparavant de la contribution de l'éducation au développement de la société? Pour répondre à cette question, l'auteur s'appuie sur la notion de crise de légitimité dans les sociétés capitalistes avancées de Habermas. Il propose une interprétation de la crise qui se situe au-delà des explications «faciles » qui renvoient à la négligence des parents, à l'incapacité de l'école de rendre des comptes, aux aptitudes déficientes des élèves. Le débat doit, selon lui, situer cette crise dans la dynamique des sociétés capitalistes avancées.

Un article de Forquin (1984) sur la sociologie du curriculum reconstitue des éléments de ce qu'on a appelé la "nouvelle " sociologie de l'éducation, ce courant de pensée qui s'est développé au sein de la discipline au cours des années soixante-dix principalement en Grande-Bretagne. L'auteur ne manque pas de souligner le caractère hétérogène du mouvement. Les enjeux sociaux de la scolarisation y sont analysés dans une perspective phénoménologique qui à la fois s'inspire d'une sociologie de la connaissance et emprunte à l'école de l'interaction symbolique. Cette approche accorde ainsi une place importante à une analyse micro-sociologique de l'éducation que la sociologie de l'éducation des années soixante avait quelque peu négligée.

Hammersley (1984) suggère un ensemble de réflexions sur les tendances micro-sociologiques et macro-sociologiques qui, avec la montée de la "nouvelle» sociologie de l'éducation et le développement des théories de la reproduction, s'affrontent au sein de la discipline. L'auteur estime que la recherche d'une solution en vue de surmonter ces tendances contradictoires a plus de chances d'être trouvée à partir d'une approche qui met l'accent sur les problèmes de recherche comme tels que sur des perspectives théoriques proprement dites.

Les lecteurs qui s'interrogent sur les tendances actuelles de la sociologie de l'éducation au Québec trouveront dans l'article de Dandurand (1984) des éléments leur permettant de se faire une idée de l'état actuel et de l'évolution de la sociologie de l'éducation au cours des dernières années. L'auteur constate que l'accessibilité de l'enseignement, les relations éducation-emploi, la culture des jeunes, la gestion de l'appareil scolaire et les enseignants ont constitué des aires traditionnelles de recherche. De plus, les grands surveys ont constitué jusqu'à récemment la principale orientation méthodologique. Il observe maintenant que de nouvelles pratiques commencent à émerger. Plus de recherches qualitatives sont réalisées. L'auteur note en outre que plusieurs sociologues de l'éducation québécois s'intéressent à la "nouvelle" sociologie de l'éducation.

\section{Les travaux ayant une portée bistorique}

Les nombreux articles ayant une portée historique qui ont été publiés au 
cours de l'année témoignent d'une nouvelle tendance au sein de la discipline. Tout se passe comme si l'invitation pressante de plusieurs sociologues comme Halsey et Karabel (1977) et Petitat (1982) en vue d'accorder plus d'importance à ce type de travaux commençait à être entendue.

Certains de ces travaux ont trait au rôle du changement technologique dans l'expansion du système d'enseignement. On se rappellera la controverse qui entoure cette question. Le système d'enseignement aurait-il connu l'expansion qu'on a observée dans la plupart des pays au cours des dernières décennies si elle n'avait pas été requise par le développement économique, si on n'avait pas eu besoin d'une main-d'œuvre de plus en plus qualifiée pour l'assurer? Ou l'expansion de l'enseignement obéit-elle à une autre dynamique, celle qui consiste pour les individus à améliorer ou à maintenir leur statut social au moyen d'une scolarité accrue? Rubinson et Ralph (1984) posent le problème de la place du changement technologique dans l'expansion de l'enseignement secondaire et post-secondaire de 1890 à 1970 aux États-Unis. Les auteurs discutent de l'importance relative du changement technologique et de la lutte des groupes en vue de s'assurer un statut en s'appuyant sur une scolarité dans l'explication de l'expansion du système d'enseignement américain.

Walters (1984) discute aussi de l'expansion de l'enseignement secondaire et post-secondaire aux États-Unis de 1922 à 1979. Il observe certes que les inscriptions dans le système scolaire ont varié directement en fonction de la demande sur le marché du travail. Les étudiants ont ainsi eu tendance à utiliser la scolarité comme un moyen en vue de se préparer à l'exercice d'occupations qu'ils anticipaient obtenir sur le marché du travail. Il estime cependant que cette explication ne peut être que partielle. Il ressort de son analyse que la fréquentation scolaire peut aussi représenter pour les jeunes une alternative à l'exercice d'une occupation lorsque le marché du travail est saturé, en attendant de se trouver un emploi. Dans cette conjoncture, la fréquentation scolaire ou l'expansion du système d'enseignement n'apparaît pas liée exclusivement aux besoins du marché du travail, et à la nécessité pour les jeunes de s'y préparer en se donnant une formation scolaire.

D'autres travaux de type historique portent sur le problème de l'égalité des chances en éducation. L'expansion et le développement du système scolaire se sontils traduits par une réduction des inégalités face à l'éducation? À partir d'une analyse de la relation entre le niveau de scolarité et l'origine sociale en France de 1874 à 1954, Garnier et Raffalovich (1984) concluent que l'expansion du système d'enseignement n'a eu que peu d'effets sur les inégalités d'accès à l'éducation au cours de cette période. Guppy, Mikicich et Pendakur (1984) arrivent sensiblement à la même conclusion dans l'étude qu'ils ont effectuée à partir d'une cohorte d'individus ayant participé à l'enquête sur la mobilité au Canada en 1973. L'origine sociale continue d'exercer une grande influence sur la fréquentation scolaire même s'il y a des indications à l'effet que celle-ci diminue faiblement avec les années. 


\section{Femmes et éducation}

Nous avons déjà été à même de constater dans les articles que nous avons sélectionnés sur les facteurs sociaux de l'apprentissage et la socialisation l'importance qu'on accorde à la variable sexe dans plusieurs de ces études. Ce phénomène n'est pas nouveau, mais il ne fait pas de doute que le féminisme contribue aussi à inspirer plusieurs travaux de sociologie de l'éducation. Nous avons pensé regrouper certains articles sous le thème "Femmes et éducation" pour mieux rendre compte de cette préoccupation au sein de la discipline. Sur le plan théorique d'abord, il y a lieu de mentionner l'article de O'Brien (1984). L'auteure montre avec vigueur que l'analyse des relations entre homme et femme et de l'éducation des femmes a été négligée dans la sociologie de l'éducation d'inspiration marxiste. Cette carence n'est pas due, selon elle, à un préjugé qui s'enracinerait dans des attitudes patriarcales chez les théoriciens en cause. Elle découlerait plutôt de ce qu'elle identifie comme des défauts sérieux des théories marxistes. Celles-ci ne tiennent pas compte de l'infrastructure de la reproduction de l'espèce, ni de la distorsion et des contradictions qui existent entre celle-ci et l'infrastructure économique.

D'autres articles portent sur l'orientation des femmes à l'intérieur du système d'enseignement et sur la place qu'elles ne parviennent pas à occuper dans la hiérarchie scolaire. Lang (1984) pose plus particulièrement le problème de l'accès aux études avancées. Son étude ne porte pas uniquement sur l'accès des femmes à ce niveau d'enseignement, mais inclut aussi d'autres groupes. Selon les idéologies scolaires et certaines théories, l'accès aux études avancées dépend essentiellement du mérite et des habilités intellectuelles des étudiants. L'auteur montre que l'accès aux études avancées n'est pas basé uniquement sur le mérite. Les résultats scolaires que les étudiants ont obtenu au niveau sous-gradué jouent certes un rôle déterminant dans l'accès à des études avancées. Mais à résultats scolaires égaux, les femmes et les étudiants issus des classes inférieures ont moins de chance d'être admis à des études supérieures, et qui plus est, de poursuivre leurs études dans les facultés d'études supérieures les plus prestigieuses.

La sous-représentation des filles dans les facultés des sciences est un problème qui retient de plus en plus l'attention parce que les facultés des sciences comptent parmi les plus prestigieuses, sont susceptibles de déboucher sur des carrières qui offrent beaucoup d'opportunités dans le contexte du virage technologique, et que les femmes risquent ainsi d'être exclues de domaines stratégiques et d'activités hautement valorisées. Ware, Steckler et Leserman (1985) proposent des éléments d'explication de ce phénomène à partir d'une recherche empirique effectuée auprès d'un groupe d'hommes et de femmes inscrits dans des départements ou des facultés des sciences aux États-Unis. Whyte (1984), pour sa part, rend compte d'une recherche action dont l'objectif était a) d'observer les stéréotypes qui caractérisent le comportement des étudiants et des professeurs dans les laboratoires et les ateliers de science et de technologie dans une école de niveau secondaire britannique, et b) de réduire le taux d'échecs dans ces matières. L'auteur discute de ses interventions en 
vue de rendre les enseignants conscients des différences qui caractérisent le comportement qu'ils ont avec les garçons et les filles dans ces activités pédagogiques.

\section{L'insertion socio-professionnelle des jeunes}

L'intégration des jeunes au milieu du travail est un problème épineux auquel la plupart des sociétés sont actuellement confrontées. Le problème se pose dans le contexte de la crise économique actuelle et des mutations technologiques et sociales qui, au-delà de la conjoncture immédiate, risquent de provoquer une redéfinition des savoirs, des compétences et des stratégies de formation. Il se traduit par un taux de chômage très élevé des jeunes et par des difficultés multiples dans leur processus d'insertion socio-professionnelle. Il n'est donc pas étonnant que les sociologues de l'éducation aient tendance, beaucoup plus que par le passé, à entreprendre des études sur le processus d'insertion socio-professionnelle des jeunes.

L'article de Marini (1984) pose le problème en le replaçant dans le contexte plus large de la transition de l'adolescence à l'âge adulte. L'auteur discute des étapes au cours desquelles l'adolescent passe d'une situation de dépendance économique et de participation à sa famille d'origine à celle de l'établissement de sa propre famille. L'intérêt de l'article tient à ce que l'analyse du processus d'insertion sociale de l'adolescent n'est pas limitée à celle de son insertion professionnelle. L'auteur l'analyse principalement du point de vue de la modification des rôles que l'adolescent a à assumer. À partir de données recueillies auprès de garçons et de filles interviewés à deux reprises (1957-1958 et 1973-1974) dans le cadre d'une étude longitudinale, l'auteur essaie de reconstituer l'ordre des événements qui ont marqué leur transition de l'adolescence à l'âge adulte, et de discuter des éléments susceptibles d'avoir influencé l'ordre dans lequel ils ont passé des rôles d'étudiant et de soldat à ceux de travailleur, de conjoint et de parent.

L'article de Laflamme (1984) est plus spécifiquement centré sur le phénomène de l'insertion professionnelle des jeunes. L'objectif de l'auteur est de proposer un cadre de référence pour une telle étude. Il commence par discuter des limites des théories économiques susceptibles de projeter un éclairage sur ce processus. Il propose ensuite une approche sociologique du phénomène axée sur deux composantes. Selon lui, on peut le définir d'une part comme un processus de socialisation lorsqu'on l'analyse du point de vue du système d'enseignement qui véhicule des informations sur le marché du travail, le développement technologique et le syndicalisme. On peut privilégier, d'autre part, les faits d'organisation lorsqu'on le situe par rapport au monde du travail et de l'entreprise qui accueille le jeune travailleur. Celle-ci se situe à un niveau donné de développement technique et de syndicalisation dans le contexte d'un marché du travail particulier.

Raffe (1984) analyse, à partir de données recueillies par sondage, la transition d'un groupe d'étudiants écossais qui quittent l'école secondaire pour entrer sur le marché du travail au cours de la crise qui a marqué le début des années quatre-vingt. 
Outre le taux de chômage, l'auteur analyse les tendances qui se manifestent dans la répartition des jeunes par industrie et par occupation, dans l'état du marché du travail, et discute du rôle des programmes spéciaux d'emploi. L'auteur remet en question plusieurs des idées reçues à ce sujet. Le chômage des jeunes n'apparaît associé ni à l'instabilité des jeunes ni à celle de la structure d'emploi. Le chômage n'aurait pas fondamentalement changé la fonction sélective de l'école. Le niveau de scolarité ou le type de diplôme détenu demeure important pour l'obtention d'un emploi. Le taux de chômage des jeunes apparaît davantage lié à la conjoncture, à la récession qu'à des changements plus profonds qui auraient modifié la structure des occupations.

Lee et Wrench (1984) posent aussi le problème que rencontrent les jeunes de seize à dix-huit ans qui quittent l'école dans le contexte de la crise actuelle, mais dans une perspective différente de celle de Raffe, et à partir de données recueillies dans le cadre d'une étude longitudinale portant sur des garçons et des filles appartenant à une minorité ethnique en Angleterre. Il ressort de leur analyse que des facteurs qui n'ont rien à voir avec le mérite ou la qualification peuvent avoir un impact sur la transition des jeunes de l'école au marché du travail: le fait d'être né avec le "bon" sexe, d'avoir la «bonne» couleur et d'appartenir à une «bonne» famille (celle qui a des «relations") peuvent s'avérer déterminant.

La transition de l'école au travail ne s'effectue pas uniquement au moment où l'étudiant a quitté l'école. Elle peut s'amorcer pendant que celui-ci la fréquente encore. C'est précisément le phénomène que D'Amico (1984) analyse en se demandant si cette expérience nuit à la réussite scolaire des étudiants. L'analyse a été conduite à partir de données recueillies dans le cadre d'une étude longitudinale portant sur des jeunes Américains. Il ressort que, dans certains groupes, le fait de travailler tout en fréquentant l'école a peu d'effets sur le rang de l'étudiant dans sa classe, même si le temps qu'il consacre à l'étude et aux loisirs, lorsqu'il travaille, diminue. Chez d'autres groupes d'étudiants cependant le fait de travailler intensément parallèlement à leurs études peut contribuer à augmenter le taux d'abandon. L'auteur se demande, dans le cas de ceux pour qui le fait de travailler parallèlement à leurs études ne se traduit pas par une diminution de leur réussite, s'il n'y a pas une correspondance entre les traits de personnalité que les employeurs recherchent et récompensent chez leurs employés et ceux que les enseignants valorisent à l'école.

Il y a lieu de porter à l'attention des lecteurs qui s'intéressent à ce thème des numéros spéciaux publiés par deux revues. La revue Recherche sociale a publié un numéro thématique (no 9, 1984) intitulé Nouvelle technologie et formation professionnelle. Ce numéro, dont la préparation a été confiée à F. Aballea et A. Glogowski, traite du problème des exigences et des besoins de formation. Il s'agit d'une part des exigences de l'entreprise en relation avec les nouvelles technologies, la qualification et la formation, et d'autre part des besoins des individus en relation avec les stratégies professionnelles et les stratégies de formation. Le deuxième est celui de la revue 
Documentation et information pédagogiques sur L'enseignement technique et professionnel (no 228, $3^{\text {e }}$ trimestre 1983). Il consiste principalement en une bibliographie commentée portant sur les thèmes suivants: a) tendance générale et direction de l'innovation dans l'enseignement technique et professionnel, b) politique, planification et administration de l'enseignement professionnel, c) rapport entre l'enseignement technique et professionnel et l'enseignement général, d) l'enseignement technique et professionnel au service du travail.

\section{L'analyse de phénomènes internes à l'institution scolaire}

Les deux principaux paradigmes (paradigme fonctionnel et paradigme radical) qui ont orienté les travaux de sociologie de l'éducation au cours de la dernière décennie ont souvent été l'objet d'une même critique. On leur a reproché de considérer le système d'enseignement comme une "boîte noire", de ne pas vraiment proposer des instruments conceptuels pour analyser la dynamique organisationnelle du système d'enseignement et les processus sociaux inhérents à l'organisation de l'école et de la classe. Plusieurs articles témoignent d'études et de recherches qui ont pour objectif de jeter un éclairage sur ces processus.

Dans un article de portée théorique, Delcourt (1984) essaie précisément de poser le problème. Selon lui, la sociologie de l'éducation "traditionnelle" a accordé beaucoup d'importance aux recherches sur la stratification, la mobilité sociale et l'impact de l'origine sociale sur les cheminements scolaires des étudiants. La "nouvelle» sociologie met davantage l'accent sur la transmission et l'acquisition de savoirs et d'informations. Elle met l'accent sur la sélection et la distribution des savoirs, sur l'analyse de la relation éducative, des programmes, et de l'organisation scolaire. On en vient alors à accorder à l'analyse de phénomènes internes à l'institution scolaire toute l'importance qu'elle devrait avoir dans l'étude de la contribution de l'éducation à la reproduction ou à la transformation de la société.

Quels moyens l'organisation scolaire met-elle en œuvre pour amener ses membres à se conformer aux objectifs de l'école? Telle est la question à laquelle Nizet (1984) essaie d'apporter des éléments de réponse en s'appuyant sur des données recueillies par entrevues auprès d'enseignants du secondaire et d'élèves de l'enseignement technique et professionnel en Belgique. L'auteur commence par reconstituer le rapport de l'élève à l'école. Deux types de rapports sont identifiés selon que la relation de l'élève à l'école est caractérisée par un décalage ou une convergence entre les projets de l'élève et les objectifs de l'école, par un contrôle fondé sur la coercition ou sur la qualité de la relation, et par une attitude de retrait ou d'implication de l'élève. Il analyse ensuite le rapport des enseignants à l'école. Deux types de rapports sont, comme dans le cas des élèves, identifiés selon que la relation de l'enseignant à l'école est caractérisée par un décalage ou une convergence entre les projets des enseignants et les objectifs de l'école, l'importance ou la faiblesse des contrôles formels, et une attitude de retrait ou d'implication des enseignants. L'auteur s'interroge enfin sur les éléments qui, au-delà du contexte organisationnel, 
pourrait expliquer l'apparition de ces décalages dans la dynamique de l'organisation scolaire.

Paty (1984), pour sa part, présente un résumé des résultats d'une recherche effectuée dans une douzaine de collèges français sur la socialisation des adolescents et plus particulièrement sur les modes de relations entre individus (élèves et adultes) de ces établissements. L'auteur a) reconstitue les " construits relationnels " des élèves et leurs attitudes par rapport à leur situation dans le collège (acceptation ou rejet du collège, sympathie ou hostilité envers les enseignants, individualisme ou solidarité entre élèves, tension ou conflit entre élèves), b) commente les grandes tendances de l'évolution des élèves entre leur entrée et leur sortie de l'établissement, de même que l'effet de certaines variables (sexe, origine sociale, expérience scolaire) sur celle-ci, et c) discute des stratégies des enseignants appelés à remplir une fonction de médiateur entre les élèves et le monde extérieur au collège.

Dans quelle mesure les enseignants qui, dans l'école, occupent une position de subordonné, détiennent-ils un pouvoir dans les organisations scolaires? La question paraît à première vue saugrenue tant on est habitué dans les études sur le pouvoir dans les organisations à mettre l'accent sur les détenteurs du pouvoir plutôt que sur les subordonnés. C'est en renversant cette perspective d'analyse que Barnett (1984) en est venu à analyser le pouvoir que peuvent détenir les enseignants dans le système d'enseignement. L'auteur construit un modèle selon lequel le pouvoir de l'enseignant réside dans l'accès qu'il ou qu'elle a à diverses personnes, diverses informations et ressources matérielles. Les administrateurs dépendent, dans une certaine mesure, de ces informations et de ces ressources, et les enseignants sont ainsi dans une position qui leur permet d'influencer le comportement de leurs supérieurs. L'auteur soumet ce modèle à la vérification à partir de données qualitatives et quantitatives recueillies auprès du personnel de trois écoles secondaires américaines. Il discute des conditions et des circonstances dans lesquelles des enseignants peuvent devenir des "chiens de garde" de l'information à cause de la place qu'ils occupent dans le réseau de communication, et détenir ainsi un certain pouvoir.

Les trois articles qui précèdent ont trait à des stratégies des élèves ou des agents du système scolaire, et illustrent bien le type d'étude qui porte actuellement sur la dynamique interne des institutions scolaires. Mais, on n'a pas cessé pour autant de s'intéresser aussi aux structures formelles des organisations scolaires et de l'école secondaire en particulier. Celles-ci ont été au cœur de plusieurs réformes de l'enseignement au cours des vingt dernières années. Un numéro thématique de l'Oxford Review of Education (no 10,1) sur les écoles secondaires en Angleterre présente un intérêt certain à cet égard. On y discute des résultats de cinq études qui ont porté sur les standard, les critères d'évaluation de la réussite des étudiants au niveau secondaire, de même que des relations qui existent entre ces critères et les ressources dont les écoles disposent. Les articles qui y sont présentés témoignent du débat qui a cours sur la qualité de l'enseignement dans les écoles secondaires polyvalentes et dans des 
écoles plus "sélectives». Divers points de vue sont exprimés en ce qui concerne la question de l'heure: jusqu'à quel point la qualité de l'enseignement a-t-elle diminué avec la mise en place d'écoles polyvalentes? On y pose le problème de l'impact des différentes formes d'organisation qui existent entre les écoles appartenant à différentes autorités locales sur les cheminements scolaires des études. On y discute en outre de la méthodologie des études comparatives pour circonscrire l'effet des différences qui existent entre les écoles sur la réussite scolaire des élèves. Les lecteurs intéressés par les problèmes relatifs à la qualité et l'efficacité de l'école secondaire, de même que par l'impact des structures scolaires de niveau secondaire sur la réussite des élèves trouveront dans ce numéro thématique une source de réflexion des plus stimulantes.

\section{Les savoirs scolaires et les programmes d'étude}

La "nouvelle " sociologie de l'éducation a proposé depuis plus d'une décennie de faire des problèmes de la sélection, de la transmission et de la gestion des savoirs au sein du système d'enseignement une priorité. Peu d'articles ont été publiés en relation avec ce thème. Les deux que nous commenterons l'ont été dans le British Journal of Sociology of Education. Il n'y a pas lieu de s'en étonner puisque c'est d'abord en Angleterre que la "nouvelle» sociologie s'est développée comme mouvement de pensée au sein de la discipline. Il est permis de se demander cependant si l'absence d'article sur cette question dans d'autres revues tient au fait que les sociologues de l'éducation n'entreprennent pas ou peu d'études dans ce domaine ou au fait que les comités de lecture ne le reconnaissent pas encore comme prioritaire.

Taylor (1984) présente les résultats d'une étude de cas sur le programme "d'études commerciales" (commerce et secrétariat) dans une école secondaire d'Australie, du point de vue de son organisation, de son contenu, de la méthodologie qu'il privilégie et des valeurs générales qu'il véhicule. La perspective d'analyse que l'auteure développe pour analyser ce programme lui permet de tenir compte de ses aspects formels et informels, explicites et implicites, des relations sociales auxquelles il donne lieu, de même que de son contenu. Cette analyse est replacée dans le contexte plus large des théories de la reproduction. L'auteure établit une correspondance entre l'idéologie transmise par ce programme d'étude et les valeurs hiérarchiques et patriarcales que les élèves retrouvent dans le milieu du travail auquel ils auront à s'intégrer. L'auteure note cependant certaines tensions dans les relations entre l'école et le milieu du travail, tensions qui pourraient faire en sorte que le processus de reproduction ne soit pas aussi complet qu'on aurait pu le penser.

McHoul et Watson (1984) analysent plus particulièrement le processus par lequel les enseignants et les élèves font appel à des notions de sens commun dans la production et la transmission des connaissances formelles de la matière enseignée. À partir d'une analyse de la conversation qui s'établit entre l'enseignant et les élèves dans une classe de géographie, les auteurs essaient de démontrer comment les 
notions de sens commun et les connaissances plus formelles véhiculées dans le programme peuvent être inter-reliées, et en quoi celles-là peuvent constituer une ressource dans la maîtrise de celles-ci.

\section{Les études ethnographiques}

Certains des travaux auxquels nous nous sommes référés jusqu'à maintenant pourraient être classés dans la catégorie des études ethnographiques. C'est le cas de certains des articles portant sur l'analyse des phénomènes internes à l'institution scolaire et sur les programmes. Nous croyons néanmoins pertinent de regrouper sous ce titre un ensemble d'articles publiés dans un numéro thématique de la revue Sociology of Education (vol. 57, no 4) pour bien refléter l'importance qu'ont prise les études ethnographiques en sociologie de l'éducation au cours des dernières années. Trois articles seront plus particulièrement portés à l'attention des lecteurs.

Lubeck (1984) analyse à partir d'une méthodologie faisant appel à l'observation, la structure du temps et de l'espace de deux classes. Il essaie de montrer en quoi celle-ci peut s'avérer une façon implicite pour les adultes de transmettre leurs valeurs aux enfants. Hargreaves (1984) analyse le processus de décision des enseignants concernant des questions académiques. À partir de l'observation de réunions d'enseignants, il montre que ceux-ci ont beaucoup plus tendance à se référer à leur expérience dans la classe qu'à des théories plus formelles, des résultats de recherches ou des études de cas. Selon l'auteur, cette tendance des enseignants d'une part les place dans une position de faiblesse par rapport à l'administration lorsque vient le temps de discuter de problèmes scolaires qui dépassent le cadre immédiat de la classe, et d'autre part, rend plus difficile l'implantation d'innovation. Celle-ci exige que les enseignants soient capables de se définir autrement qu'en fonction de leur pratique et de leur expérience immédiate.

Finley (1984) analyse le contexte institutionnel dans lequel s'effectue le classement des élèves, et plus spécifiquement leur répartition dans quatre groupes homogènes correspondant à des niveaux d'habilité différents en anglais (langue maternelle) dans une école secondaire polyvalente américaine. L'étude est centrée sur les stratégies des enseignants face au classement, et a été effectuée à partir de données recueillies par entrevues et observation participante. Il ressort a) que les enseignants préferent dispenser leur enseignement aux groupes d'élèves les plus forts qui aspirent à des études post-secondaires, b) que le fait d'enseigner à ces groupes est perçu par les enseignants comme une récompense et un indicateur du statut dont ils bénéficient dans la hiérarchie de l'enseignement, c) que leur recherche d'un statut plus élevé dans cette hiérarchie contribue à façonner et à maintenir le système de classement, d) que ce système a un impact sur leur satisfaction face à l'enseignement comme carrière et leur sentiment d'être compétent, et que, dans cette perspective, les enseignants sont eux-mêmes "classés " tout comme les élèves le sont, e) que le système de classement est structuré en fonction des besoins des enseignants autant que par ceux des étudiants. 


\section{Conclusion}

Il ressort de cette recension d'articles de revues parus en 1984 que certains domaines traditionnels de recherche continuent d'occuper une place de premier choix au sein de la discipline. C'est le cas des études sur les facteurs sociaux de l'apprentissage et sur le processus de socialisation. On aura noté aussi l'émergence de préoccupations nouvelles. Les revues ont en effet publié plusieurs articles sur des thèmes auxquels on n'aurait pas, nous semble-t-il, fait autant de place dans le passé. Qu'il suffise de mentionner les travaux ayant une portée historique, de même que les articles sur le processus d'insertion socio-professionnelle des jeunes, les femmes en éducation, les savoirs scolaires et les programmes. Sur le plan théorique, c'est le débat sur les théories de la reproduction qui monopolise l'attention. L'analyse des phénomènes internes à l'institution scolaire de même que le problème de l'articulation entre des perspectives macro-sociologiques et micro-sociologiques apparaissent de plus en plus comme des préoccupations centrales au sein de la discipline. Sur le plan méthodologique, les revues semblent d'une part accorder une priorité à la publication de résultats de recherches longitudinales et d'autre part, consacrer plus d'espace que par le passé aux études ethnographiques.

Claude Trottier Université Laval

\section{NOTE}

1. Note de la rédaction

C'est à la suite d'une recommandation de son comité de rédaction que la Revue des sciences de l'éducation présente une recension d'articles de revues parus en 1984 dans le domaine de la sociologie de l'éducation. Le Comité de rédaction a en effet recommandé que la Revue fasse état des articles qui, en sociologie entre autres, pourraient contribuer à l'avancement des sciences de l'éducation ou au renouvellement de leurs problématiques. La Rédaction souhaite que la publication de telles recensions puisse être réalisée annuellement et pour d'autres disciplines.

\section{RÉFÉRENCES}

Alwin, D.F. et A. Thornton, Family origins and the schooling process: early versus late influence of parental characteristics, American Sociological Review, 49,6, 1984, p. 784-802.

Barnett, B.G., Subordinate teacher power in school organizations, Sociology of Education, 57,7, 1984, p. 43-55.

Close, D., Canadian students and world affairs, Canadian Journal of Education, 9,3, 1984, p. 331-342. 
Crespo, M. et G. Pelletier, Academic performance, social integration and francophone "classes d'accueil" for young immigrants: diachronic analysis of an educational experience in Montreal (1974-1983). Compare: a Journal of Comparative Education, 14,1, 1984, p. 7-19.

D'Amico, R., Does employment during high school impair academic progress, Sociology of Education, 57,3, 1984, p. $152-163$.

Dandurand, P., La recherche en sociologie de l'éducation au Québec, Prospectives, 20 1-2, 1984, p. 69-75.

Delcourt, J., Vers une nouvelle sociologie de l'éducation, Recherches sociologiques, XV, 1, 1984, p. 11-28.

Documentation et Information Pédagogiques, numéro sur le thème L'enseignement Technique et professionnel no 228 ( $3^{\mathrm{e}}$ trimestre), 1983.

Dubar, C., Les fonctions de la formation continue en France: interprétations et confrontations théoriques, Recherches Sociologiques, XV, 1, 1984, p. 29-36.

Educational Review, Special Issue (16). Political Education in 1984, vol. 36, no 2, 1984.

Falsey, B. et B. Heyns, The college channel: private and public schools reconsidered, Sociology of Education, 57,2, 1984, p. 111-121.

Finley, M., Teachers and tracking in a comprehensive high school, Sociology of Education, 57,4, 1984, p. 233-243.

Forquin, J.C., La sociologie du curriculum en Grande-Bretagne: une nouvelle approche des enjeux sociaux de la scolarisation, Revue Française de Sociologie, XXV, 2, 1984, p. 211-232.

Garnier, M.A. et L.E. Raffalovich, The evolution of equality of educational opportunities in France, Sociology of Education, 57,1, 1984, p. 1-10.

Gordon, L., Paul Willis - Education, cultural production and social reproduction, British Journal of Sociology of Education, 5,2, 1984, p. 105-115.

Guppy, N., P.D. Mikicich et R. Pendakur, Changing patterns of educational inequality in Canada, Cabiers Canadiens de Sociologie, 9,3, 1984, p. 319-332.

Halsey, A.H. et J. Karabel, Power and Ideology in Education, New-York: Oxford University Press, 1977.

Hammersley, M., Some reflections upon the macro-micro problem in the sociology of education, The Sociological Review, 32,2, 1984, p. 316-325.

Harber, C.R., Development and political attitudes: the role of schooling in Northern Nigeria, Comparative Education, $20,3,1984$, p. $387-403$.

Hargreaves, A., Experience counts, theory doesn't: how teachers talk about their work, Sociology of Education, 57,4, 1984, p. 244-253.

Harker, R.K., On reproduction, habitus and education, British Journal of Sociology of Education, 5,2 1984, p. 117-127.

Hearn, J.C., The relative roles of academic, ascribed and socio-economic characteristics in college destinations, Sociology of Education, 57,1, 1984, p. 22-30.

Jackman, M.R. et M.J. Muha, Education and intergroup attitudes: moral enlightenment, superficial democratic commitment, or ideological refinement?, American Sociological Review, 49,6, 1984, p. 751-769.

Laflamme, C., Une contribution à un cadre théorique sur l'insertion professionnelle des jeunes, Revue des sciences de l'éducation, X, 2, 1984, p. 119-216.

Lang, D., Education, stratification and the academic hierarchy, Research in Higher Education, 21,3, 1984, p. $329-352$.

Lee, G. et J. Wrench, 16-18: The crisis of the school leaver, Culture, Education and Society, 38,4, 1984, p. 321-347.

Lubeck, S., Kinship and classroom: an ethnographic perspective on education as cultural transmission, Sociology of Education, 57,4, 1984, p. 219-232.

Marini, M.M., The order of events in the transition to adulthood, Sociology of Education, 57,2, 1984, p. 63-83.

McHoul, A.W. et D.R. Watson, Two axes for the analyses of "commonsense" and "formal" knowledge in classroom talk, British Journal of Sociology of Education, 5,3, 1984, p. 281-302.

Ninalowo, B., Education, legitimation and crisis, Canadian Journal of Education, 9,3, 1984, p. 298-316.

Nizet, J., L'exercice du contrôle dans les écoles secondaires, Recherches sociologiques, XV, 1, 1984, p. 67-91.

O'Brien, M., The commatization of women: patriarchal fetishism in the sociology of education, Interchange, 15,2, 1984 , p. $43-60$. 
Oxford Review of Education, Special Issue. Comprehensive and Selective Schooling, 10,1, 1984.

Passeron, J.-C. et F. De Singly, Différences dans la différence: socialisation de classe et socialisation sexuelle, Revue Française de Science Politique, 34,1, 1984, p. 48-78.

Paty, D., Socialisation des adolescents et organisation scolaire. Étude du fonctionnement des collèges français, Recherches sociologiques, XV, 1, 1984, p. 58-66.

Petitat, A., Production de l'École. Production de la société. Analyse socio-bistorique de quelques moments décisifs de l'évolution scolaire de l'Occident. Genève: Librairie Droz, 1982.

Raffe, D., The transition from school to work and the recession: evidence from the scottish school leavers surveys, 1977-1983. British Journal of Sociology of Education, 5,3, 1984, p. 247-265.

Recherche Sociale, Numéro sur le thème "Nouvelle Technologie et Formation Professionnelle", 91 (juillet/ septembre), 1984.

Rubinson, R. et J. Ralph, Technical change and the expansion of schooling in the United States, 1890-1970, Sociology of Education, 57,3, 1984, p. 134-152.

Shapiro, S., Ideology, Hegemony, and the individualizing of instruction: the incorporation of "progressive" education, Journal of Curriculum Studies, 16,4, 1984a, p. 367-378.

Shapiro, S., Crisis of legitimation: schools, society and declining faith in education, Interchange, 15,4, 1984b, p. 26-39.

Taylor, S., Reproduction and contradictions in schooling: the case of commercial studies, British Journal of Sociology of Education, 5,1, 1984, p. 3-18.

Walters, P.B., Occupational and labor market effects on secondary and postsecondary educational expansion in the United States: 1922 to 1979, American Sociological Review, 49,5, 1984, p. 659-671.

Ware, N., N.A. Steckler et J. Leserman, Undergraduate Women: who chooses a science major. The Journal of Higher Education, janvier, février 1985, p. 73-84.

Whyte, J., Observing sex stereotypes and interactions in the school lab and workshop, Educational Review, 36,1, 1984, p. 75-86.

Willis, P., Learning to labor. How Working Class Kids Get Working Class Jobs, London: Hutchinson, 1977.

Willis, P., Cultural production and theories of reproduction, in L. Barton et S. Walker (éds.), Rare, Classe and Education, London: Croom Helm, 1983, p. 107-137. 